\title{
THE MARBLL EXPERIMENT: TOWARDS A MARTIAN WIND LIDAR
}

Anni Määttänen ${ }^{1 *}$, François Ravetta ${ }^{2}$, Franck Montmessin ${ }^{1}$, Didier Bruneau ${ }^{1}$, Jean-François Mariscal $^{1}$, Mathilde Van Haecke ${ }^{1}$, Guillaume Fayolle ${ }^{1}$, Christophe Montaron ${ }^{1}$, David Coscia ${ }^{1}$

\author{
${ }^{1}$ LATMOS/IPSL, UVSQ Université Paris-Saclay, UPMC Univ. Paris 06, CNRS, \\ Guyancourt, France, *anni.maattanen@latmos.ipsl.fr \\ ${ }^{2}$ LATMOS/IPSL, UPMC Univ. Paris 06 Sorbonne Universités, UVSQ, CNRS,
}

\section{Paris, France}

\begin{abstract}
Operating a lidar on Mars would fulfill the need of accessing wind and aerosol profiles in the atmospheric boundary layer. This is the purpose of the MARs Boundary Layer Lidar (MARBLL) instrument. We report recent developments of this compact direct-detection wind lidar designed to operate from the surface of Mars. A new laser source has been developed and an azimuthal scanning capability has been added. Preliminary results of a field campaign are presented.
\end{abstract}

\section{INTRODUCTION}

The LATMOS laboratory has been developing since 2008 a compact and robust direct-detection wind lidar based on a Mach-Zehnder interferometer concept and designed to operate from the surface of Mars. MARBLL uses a mature state-of-the-art wind lidar technology, including a $\mathrm{Nd}$ :Yag laser emitting at $1064 \mathrm{~nm}$. The MachZehnder interferometer is used as a spectral analyzer. The wind measurement, as for all Doppler wind lidars (DWLs), is based on measuring the Doppler shift induced by the radial velocity of aerosols backscattering laser photons to the telescope. Traditionally the DWLs use methods based on quasi-monomode laser emission and require precise frequency locking between the emitter and the analyzer. Such methods have not yet been demonstrated during a space mission. The original MARBLL design included a laser inherited from the ChemCam instrument [1] on the Mars Science Laboratory Curiosity rover. Lidars are perfectly well suited to the dusty conditions encountered on Mars, with suspended dust particles highly reflective at the laser wavelength $\left(\omega_{0}=0.98\right)$. Bruneau et al. (2013) [2] describe the instrument, the optical design and the results of the first field campaign at the OHP site (Observatoire de Haute-Provence, France).

\section{METHODOLOGY}

\subsection{Description of the campaign}

MARBLL was set up for a week at the Gérard Mégie station of the OHP site in southeastern France in September 2016. The station performs continuous geophysical measurements including atmospheric balloon radio soundings $(\mathrm{p}, \mathrm{T}, \mathrm{z}, \mathbf{V})$ and provides a platform for instrumental campaigns, such as ours. The OHP site $\left(5^{\circ} 43^{\prime} \mathrm{E}\right.$, $44^{\circ} 56^{\prime} \mathrm{N}$ ) hosts also a mast (ICOS project, www.icos-ri.eu) that provides, for example, wind measurements at three heights $(10,50$ and 100 meters) and a compact automated aerosol lidar (CE376, www.cimel.fr) working at 532 nanometers. These measurements can be compared with MARBLL winds and particle backscatter profiles.

The original Mach-Zehnder interferometer (MZI) was designed specifically to match the optical requirements of the ChemCam laser EDU 1 (the optical path of the prisms inside the MZI is aligned with the laser cavity). The new set-up of the instrument uses another laser source (for all laser parameters see Table 1). The new laser, E3M, has been conversely designed in-house to fit the parameters required by the heritage interferometer. The mechanical design includes the possibility for a motorized scan over a desired range of azimuth angles and a manual zenithal angle variation. The electronics design was modified to include a correction enabling the scanning sequence.

The campaign was focused on validating the portability of the instrument and the consequent optical mounting and re-alignment, the mechanical 
design and the electronics design that pilots and synchronizes the scan and measurement sequences.

Table 1 E3M and EDU1 laser specifications

\begin{tabular}{|l|l|l|}
\hline Laser & $\begin{array}{l}\text { E3M } \\
\text { (in-house) }\end{array}$ & $\begin{array}{l}\text { EDU1 } \\
\text { (MSL heritage) }\end{array}$ \\
\hline Crystal & Nd:YAG & Nd:KGW \\
\hline Wavelength & $1064 \mathrm{~nm}$ & $1067 \mathrm{~nm}$ \\
\hline Energy & $12,5 \mathrm{~mJ}$ & $>24 \mathrm{~mJ}$ \\
\hline Pulse duration & $4.4 \mathrm{~ns}$ & $<8 \mathrm{~ns}$ \\
\hline Repetition rate & $100 \mathrm{~Hz}$ & $1-10 \mathrm{~Hz}$ \\
\hline
\end{tabular}

\subsection{Performed measurement sequences}

We performed three types of measurements:

1. Simple zenithal or oblique measurements;

2. VAD (Velocity-Azimuth Display) scans of different durations at $45^{\circ}$ zenithal angle with different azimuthal angle steps within the $180^{\circ}$ total scan range allowed at the site;

3. DBS (Doppler Beam Swinging) measurements (3-5 azimuthal directions with different zenithal angles).

The laser was operated at $100 \mathrm{~Hz}$ frequency and the measurement integration times were varied. Postprocessing tests were made to find the optimal averaging of data: in the results presented here a 10 s averaging has been used (1000 laser shots were averaged for one profile) and data with signal-tonoise ratio $\mathrm{SNR}<10$ were discarded.

\section{RESULTS}

We present here the MARBLL measurements made with static pointing and also during a VAD sequence. Results are compared with other concomitant measurements (see below). The DBS sequences are yet to be analyzed and are therefore not presented here.

\subsection{Wind profile comparisons}

The first simple oblique measurements were performed at the beginning of the campaign (September $14^{\text {th }}$ ). Measurements were made at a $45^{\circ}$ zenith angle during 15 minutes towards North and then East. This gave us the opportunity for a first quick comparison of the MARBLL wind measurements with a radiosonde launched simultaneously and with the wind sensors of the ICOS mast. The results for the North wind component measured by MARBLL simultaneously with the radio sounding are shown in Figure 1. The comparison shows a fair agreement within the $\mathrm{ABL}$ where sufficient amounts of aerosols allow MARBLL to collect enough signal exceeding the SNR 10 threshold.

Figure 2 shows a similar measurement for the Eastward component from a measurement sequence performed two days later (September $16^{\text {th }}$, see section 3.2). The wind profiles are analyzed from measurements performed with the lidar pointing towards West at $45^{\circ}$ zenith angle for one minute. A radio sounding was launched at around the same time and allowed further comparison in addition to the ICOS data. The comparison is good overall, but the ICOS mast indicated much stronger winds at $100 \mathrm{~m}$ than both the radio sounding and MARBLL, whereas around $50 \mathrm{~m}$ the agreement between the three measurements seems to be better.

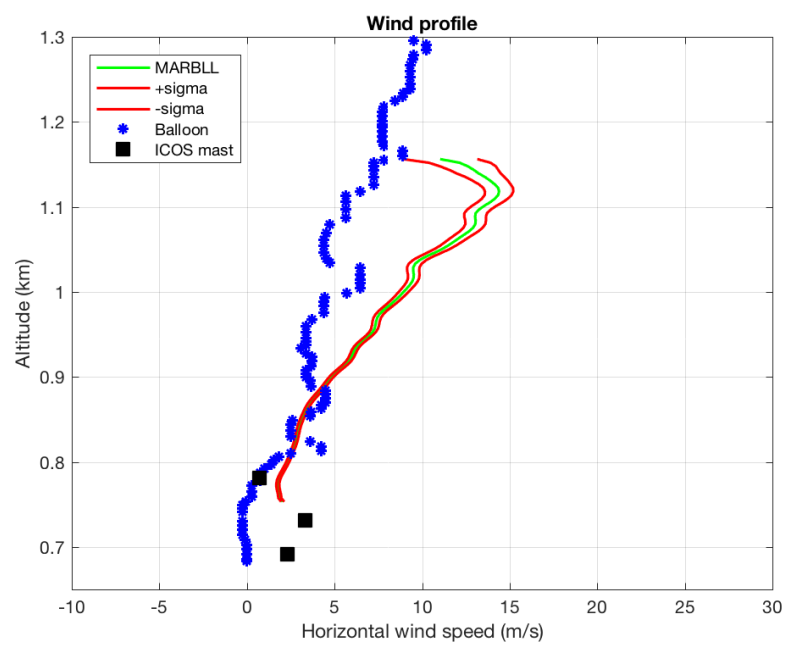

Figure 1: A comparison of the northward wind component profile measured on September 14, 2016 by MARBLL (green line) with simultaneous radio soundings (blue asterisks, smoothed over 15 points) and the ICOS anemometers (black squares). Altitude is the altitude above sea level (OHP is at $681 \mathrm{~m}$ asl). $M A R B L L$ measurements with $S N R<10$ and radial wind standard deviation $>5 \mathrm{~m} / \mathrm{s}$ are not displayed. 


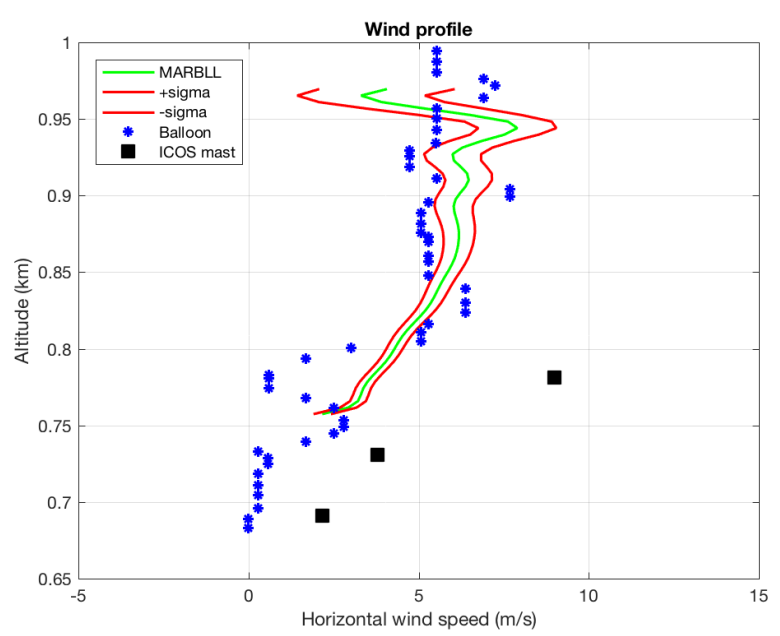

Figure 2: Same as Figure 1, but for the eastward wind component on September $16^{\text {th }}$.

Figure 3 compares the MARBLL measurements obtained $100 \mathrm{~m}$ above ground level, the same elevation as the ICOS wind sensors. The agreement is particularly convincing. Note that this plot also shows the variability of the wind field in the boundary layer. In such turbulent conditions, no perfect match can be expected when comparing MARBLL to radio soundings.

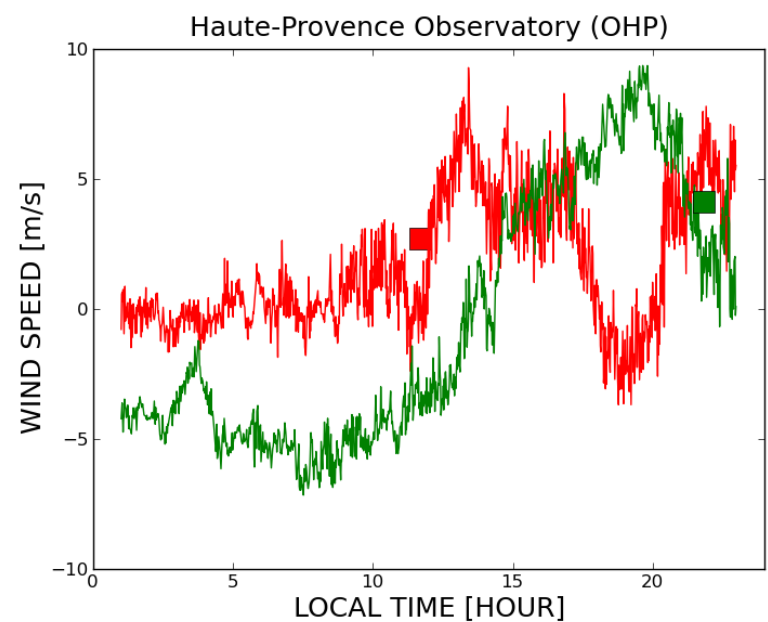

Figure 3: Horizontal wind component $(\mathrm{m} / \mathrm{s})$ as a function of time at OHP on September 14 (meridional wind in red) and on September 16 (zonal wind in green). Continuous lines are the measurements from the ICOS mast (100 $\mathrm{m}$ above ground level), squares are MARBLL measurement at the same altitude.

\section{2 $\quad 180^{\circ} \mathrm{VAD}$ sequences}

The first validated, full $180^{\circ}$ VAD sequences were performed on September $16^{\text {th }}$ with two adjacent radio soundings. During the first sequence,
MARBLL scanned the atmosphere at $45^{\circ}$ zenithal angle from West (through North) to East with $3^{\circ}$ intervals with 60 s integration time per direction. We fit the data to extract the expected sinusoidal behavior of the wind and thus access both the wind speed and direction along the vertical [3].

The VAD analysis was performed only in the very shallow aerosol-laden ABL between about $70 \mathrm{~m}$ to $520 \mathrm{~m}(0.75-1.2 \mathrm{~km}$ asl $)$. At higher altitudes, the error on individual wind measurement gets significant, but the sinusoidal behavior can still be fitted (not shown).

Wind speed and direction are shown in Figure 4 together with the radio soundings. The profiles reveal similar tendencies, but in the lowest 200 meters the balloon measurements seem perturbed by the oscillating motion of the sounder (not shown). Above about $1 \mathrm{~km}$ the MARBLL errors increase significantly. Some assumptions regarding the properties of the background wind field (both stationary and homogeneous) are intrinsically made in the VAD analysis. MARBLL VAD thus provides an average view of the wind field from which radio soundings can depart substantially given the localized nature in both space and time of their measurements.
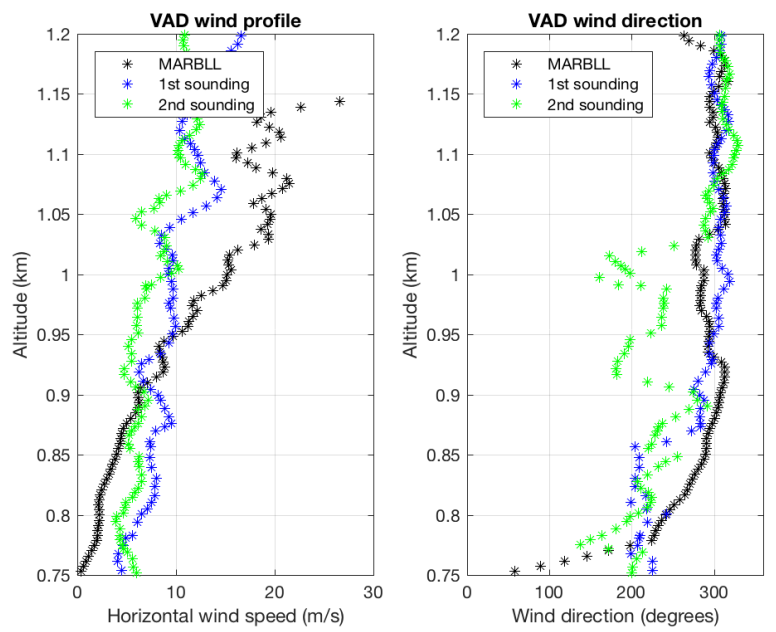

Figure 4: Profiles of wind speed (left) and direction (right) extracted from the VAD analysis and two radio soundings $\left(1^{\text {st }}\right.$ in the beginning of the MARBLL sequence, $2^{\text {nd }} 30$ minutes after the end of the sequence). MARBLL errors increase significantly above $0.95 \mathrm{~km}$.

\subsection{Aerosol layer and cloud detection}

The Phoenix lidar has been so far the only lidar on the Martian surface. Phoenix lidar made pioneering 
observations of precipitating water ice clouds in the polar atmosphere [4]. Since one of the goals on MARBLL on Mars will be cloud detection and aerosol profile measurements from the surface, the last morning of the campaign was devoted to performing a long static zenithal measurement sequence that was started slightly before sunrise and was continued during three hours to monitor the development of the boundary layer. Unfortunately, the campaign week was very rainy, which reduced greatly the aerosol concentrations. Thus, direct comparison of aerosol profile is not possible. However, clouds were detected during this sequence. Comparison of the partly precipitating cloud structures, as seen by both lidars (MARBLL on Figure 5, ICOS on Figure 6), shows great consistency.

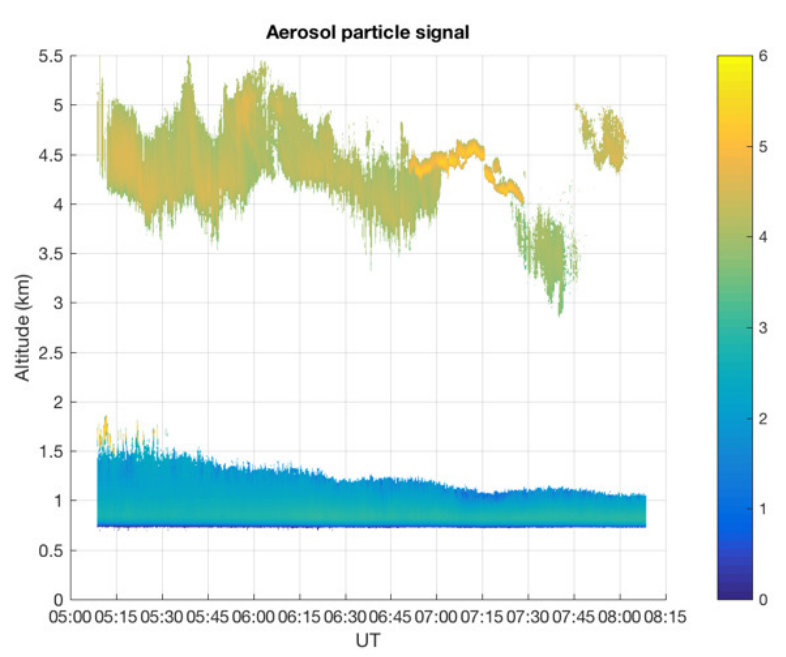

Figure 5: MARBLL range corrected signal@1064nm (arbitrary units, SNR > 10) as a function of altitude from 5 UT to 8 UT (sunrise at 5:20 UT).

\section{CONCLUSIONS}

The OHP MARBLL validation campaign suffered from the aerosol-poor conditions at the OHP site during the rainy campaign week, limiting the signal to a very shallow layer near the ground. Nevertheless, several key measurements (wind speed and direction, cloud geometry) were made and compared to radio soundings, wind measurements on a mast and aerosol lidar data. Further comparison of MARBLL with another wind profiler (radar or lidar) is foreseen. The OHP campaign allowed us to validate several key aspects of the instrument in "real" (although terrestrial) conditions: in particular, the VAD scan capability has been validated, in line with expectations made for possible Mars operations. The OHP campaign set the major milestones of the MARBLL development roadmap for 2017 and beyond. A new measurement campaign is planned in dustier conditions to better assess the instrument performances in Mars-like conditions.

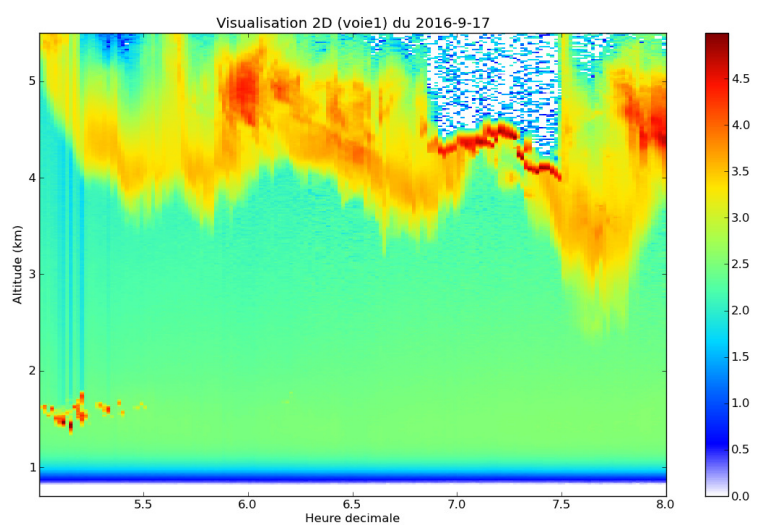

Figure 6: ICOS lidar range-corrected signal (arbitrary units)@532nmvs. altitude from 5 to 8 UT.

\section{ACKNOWLEDGEMENTS}

We are grateful to Pierre-Eric Blanc and Ilja Reiter for providing access to the ICOS data, and to the entire staff of the Gérard Mégie station for their support. We also thank the FR 3098 ECCOREV and the OSU PYTHEAS. We thank the French Space Agency CNES for their support to the development of MARBLL.

\section{References}

[1] Maurice, S., Wiens, R.C., Saccoccio, M. et al. Space Sci Rev (2012) 170: 95. doi:10.1007/s11214-012-9912-2

[2] Bruneau D., et al., Direct-detection wind lidar operating with a multimode laser, Appl. Opt. 52, 4941-4949 (2013).

[3] Werner, C., chapter 12 in "Lidar", Vol. 102, Springer Series in Optical Sciences, pp 325-354

[4] Whiteway, J. A. et al., Mars Water-Ice Clouds and Precipitation, Science 325 (5936), pp. 6870, DOI: $10.1126 /$ science.1172344. 